\title{
Glucose infusion-induced liver dysfunction following treatment of prolonged hypoglycaemia after a massive insulin overdose
}

\author{
ZAKA HAQ, ${ }^{1}$ MAMTA JOSHI, ${ }^{1}$ SONYA GHATAHORA, ${ }^{2}$ MICHAEL OKORIE2,3
}

\section{Introduction}

There has been ambiguity in managing the complications related to insulin overdose, despite the passage of about 70 years since the first case was reported. ${ }^{1}$ Mild-to-moderate, transient and self-limiting liver dysfunction associated with high strength and prolonged dextrose load to treat hypoglycaemia due to insulin overdose has been reported sparsely in the literature and is relatively poorly understood. ${ }^{2}$ We attempt to highlight this uncommon, transient complication of managing hypoglycaemia in a patient with insulin overdose.

Key words: insulin overdose, prolonged hypoglycaemia, glucose load, liver dysfunction, hepatic injury

\section{Case summary}

A 27 year old male self-presented to the Emergency Department an hour after a deliberate insulin overdose. He had injected 1,500 units of the long acting insulin analogue, glargine (Lantus ${ }^{\circledR}$ and 1,500 units of the rapid acting insulin analogue, aspart $\left(\right.$ NovoRapid $\left.^{\circledR}\right)$, with suicidal intent. The patient had not taken any other prescribed, over-the-counter or illicit drugs and there was no history of recent alcohol intake.

He had a 15 year history of type 1 diabetes which was reasonably well controlled $\left(\mathrm{HbA}_{1 \mathrm{c}} 54 \mathrm{mmol} / \mathrm{L}\right)$ with no microvascular complications. He had no previous history of deliberate self-harm or mental health illness, but mentioned that he had anxiety and heard voices in his head telling him to overdose on insulin to commit suicide. He had wheat intolerance and was not on any regular medications apart from his regular insulin. The patient was unemployed, never smoked, rarely drank alcohol and lived with his mother. On examination, he was haemodynamically and neurologically stable with a Glasgow coma scale of 15/15.

Admission capillary blood glucose was $3.1 \mathrm{mmol} / \mathrm{L}$ and other

Kings College Hospital London, London, UK

2 Brighton and Sussex University Hospitals, Brighton, UK

${ }^{3}$ Brighton and Sussex Medical School Brighton, UK

Address for correspondence: Dr Zaka Haq

Department of Diabetes, Darrent Valley Hospital Dartford DA2 8DA

E-mail: zakaulhaq2002@yahoo.com

Br J Diabetes Vasc Dis 2015;15:192-194

http://dx.doi.org/10.15277/bjdvd.2015.048
Figure 1. Graph of capillary blood glucose monitoring (over 4 days)

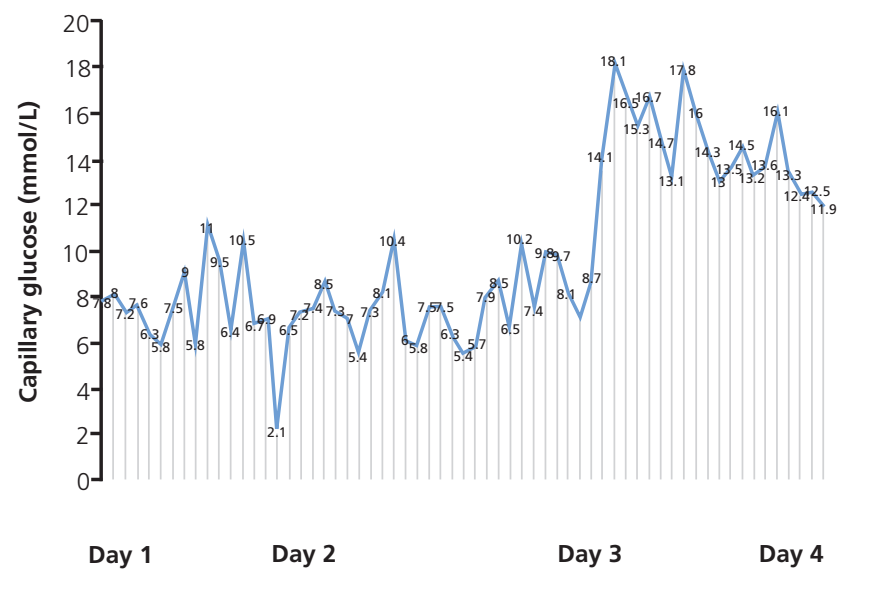

blood tests including full blood count, renal function and liver function were normal. Serum paracetamol and salicylate levels were undetectable.

In view of the large insulin overdose and hypoglycaemia on presentation, he was started on a $10 \%$ glucose infusion. Due to persistent hypoglycaemia and problems sustaining his glucose to a safer range, he was subsequently started on $20 \%$ glucose infusion, which was continued over the next four days, with frequent capillary glucose monitoring (Figure 1). He typically required $600-800 \mathrm{~g}$ of glucose per day to maintain euglycaemia. By day 4 the glucose infusion was stopped due to rising capillary glucose levels and he was started on his regular subcutaneous insulin at reduced doses.

This patient's liver function tests were noticed to be deranged on day 2 (Figure 2) but subsequent viral, autoimmune and toxic liver screens did not reveal any other abnormality. Liver ultrasound showed diffusely-raised echogenicity. Due to the unclear nature of hepatic dysfunction, the Hepatology team suggested a trial infusion of $\mathrm{N}$-acetylcysteine (NAC; see legend to Figure 2 for the protocol used).

His liver function gradually improved over the next few days and he was seen by the liaison psychiatry team and started on sertraline. He was discharged on day 6 with a serum ALT level 
Figure 2. Trends of liver function tests during the hospital admission

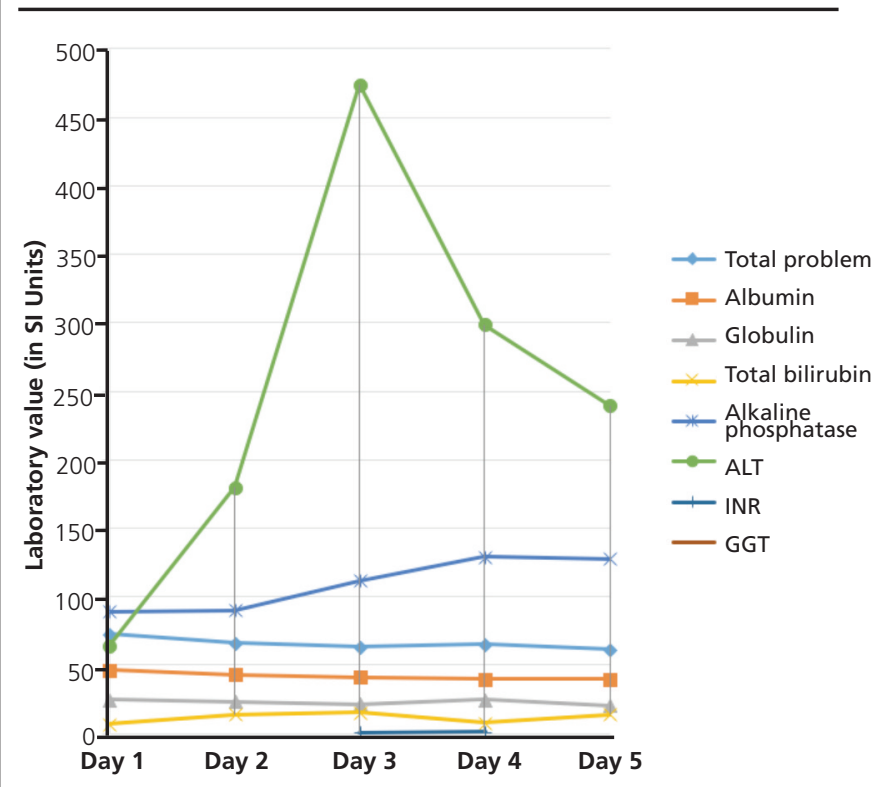

$\mathrm{N}$-Acetylcysteine (NAC) protocol: $49 \mathrm{~mL} \mathrm{NAC}$ in $200 \mathrm{~mL} 0.9 \%$ over 1 hour; $17 \mathrm{~mL}$ NAC in $500 \mathrm{~mL} 0.9 \%$ over 4 hours; $33 \mathrm{~mL}$ NAC in $1 \mathrm{~L} 0.9 \%$ over 16 hours.

of $240 \mathrm{IU} / \mathrm{L}$ (day 5), stable glucose of $11.8 \mathrm{mmol} / \mathrm{L}$ and a prescription for his regular subcutaneous insulin. Subsequently the patient was lost to follow-up but 3 months later upon contact agreed to have his liver function tests repeated which showed complete normalisation.

\section{Discussion}

Insulin overdoses are becoming a familiar presentation to accident and emergency departments within the context of an increased burden of diabetes and a rising incidence of mental health issues associated with diabetes. While the management of hypoglycaemia takes a priority to prevent neurological injury, other complications such as hypokalaemia, hypomagnesaemia, pulmonary oedema and hepatic dysfunction also need to be monitored carefully. 3,4

Glargine is a long acting peakless basal insulin which is deposited in the subcutaneous tissue in a polymeric form with gradual release for prolonged action. ${ }^{5}$ The pharmacokinetic profile of glargine can be predicted for physiologic doses, but it becomes difficult to identify the pattern and duration of release of this long acting insulin following a massive overdose. ${ }^{6}$

We identified about 15 cases of glargine overdose reported in the literature. UK national guidelines for the hospital management of hypoglycaemia recommend 150-200 mL intravenous $10 \%$ glucose, or $75-100 \mathrm{~mL} 20 \%$ glucose for obtunded patients. ${ }^{7}$ However, titration of dextrose infusions to achieve euglycaemia results in loading with large amounts of glucose for patients with prolonged hypoglycaemia, which may result in acute hepatic injury. ${ }^{8}$

\section{Key messages}

- Hepatic dysfunction associated with a glucose load after a massive insulin overdose is well known in the literature, but many frontline clinicians are unaware of it

- Specialist hepatic investigations may be reserved for patients with massive insulin overdose in whom liver function tests fail to normalise one week after their recovery from overdose

- Such an approach will avoid unnecessary investigations and might facilitate early discharge from hospital

The mechanism for hepatic injury following a glucose load is uncertain. Tsujimoto et al reported a case of rapid onset glycogen storage hepatomegaly secondary to the treatment of glargine overdose with infusion of large volumes of glucose. ${ }^{8}$ They demonstrated glycogen deposition and the resolution of the same in liver histology samples. Jolliet et al also reported a similar case of rapid onset liver dysfunction and postulated that this could have been due to acute hepatic steatosis, secondary to triglyceride accumulation. ${ }^{9}$ Tsujimoto et al showed that derangement started in 24-48 hours and recovery took more than two weeks, while the case reported by Jolliet et al showed that derangement started after day 3 with recovery in 48 hours from peak, i.e. by day $7.8,9$

We recorded rapid onset hepatic enzyme derangement in our patient, which was seen by the second day of glucose loading. With the stabilisation of hypoglycaemia and resumption of insulin therapy from day 5, we noted gradual improvement in the liver enzymes. The role of NAC in the recovery is unclear, as its use has not been reported by previous authors in similar case scenarios. As reported by Mumtaz et al, the use of NAC in nonparacetamol induced liver failure resulted in a reduction in mortality, possibly due to various uncertain mechanisms including a reduction in oxidative damage. Hence, there may have been some benefit of using NAC in our patient. ${ }^{10}$

Other strategies suggested for managing prolonged hypoglycaemia following insulin overdose include surgical excision of the depot site, the use of glucocorticoids to induce insulin resistance and the use of glucagon to alleviate the need for glucose loading.2,4 The obvious dearth of reported literature and limited knowledge in this area can lead to unnecessary investigations, anxiety for the patient and also prolonged hospital length of stay.

\section{Conclusion}

An awareness of the phenomenon of hepatic dysfunction related to glucose infusions following an insulin overdose might enable doctors treating such patients to avoid unnecessary liver 
investigations. It must be stated, however, that other aetiological factors should be considered if there is a delay in the recovery of hepatic function.

\section{Conflict of interest None \\ Funding sources None}

\section{References}

1. Jordan H. Unusual sequel of large overdose of insulin. BMJ 1946;1:276. http://dx.doi.org/10.1136/bmj.1.4442.276

2. Eldred AE, Mustafa OG, Hunt KF, Whitelaw C. Problem based review: the patient who has taken an overdose of long-acting insulin analogue. Acute Med 2013;12:167-72.

3. Tofade TS, Liles EA. Intentional overdose with insulin glargine and insulin aspart. Pharmacotherapy 2004:24:1412-18.

http://dx.doi.org/10.1592/phco.24.14.1412.43147

4. Warriner D, Debono M, Gandhi RA, et al. Acute hepatic injury following treatment of a long-acting insulin analogue overdose necessitating urgent insulin depot excision. Diabet Med 2012:29:232-5. http://dx.doi.org/10.1111/j.1464-5491.2011.03385.x
5. Gillies PS, Figgitt DP, Lamb HM. Insulin glargine. Drugs 2000;59:253-60. http://dx.doi.org/10.2165/00003495-200059020-00009

6. Groth CM, Banzon ER. Octreotide for the treatment of hypoglycemia after insulin glargine overdose. J Emerg Med 2013;45:194-8. http://dx.doi.org/10.1016/j.jemermed.2012.11.099

7. Joint British Diabetes Societies. The Hospital Management of Hypoglycaemia in Adults with Diabetes Mellitus. Revised September 2013. Available from: http://www.diabetologists-abcd.org.uk/subsite/JBDS_IP_ Hypo_Adults_Revised.pdf.

8. Tsujimoto T, Takano M, Nishiofuku M, et al. Rapid onset of glycogen storage hepatomegaly in a type- 2 diabetic patient after a massive dose of long-acting insulin and large doses of glucose. Intern Med 2006;45:46973. http://dx.doi.org/10.2169/internalmedicine.45.1548

9. Jolliet $\mathrm{P}$, Leverve $X$, Pichard C. Acute hepatic steatosis complicating massive insulin overdose and excessive glucose administration. Intensive Care Med 2001;27:313-16. http://dx.doi.org/10.1007/s001340000763

10. Mumtaz K, Azam Z, Hamid S, et al. Role of N-acetylcysteine in adults with non-acetaminophen-induced acute liver failure in a center without the facility of liver transplantation. Hepatol Int 2009:3:563-70. http://dx.doi.org/10.1007/s12072-009-9151-0

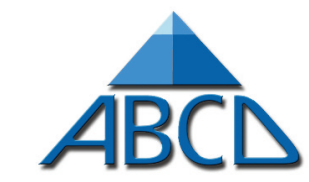

Association of British Clinical Diabetologists

\section{Exenatide weekly (QW) (Bydureon) Nationwide Audit in progress}

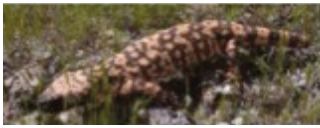

\section{Does your centre use exenatide QW?}

\section{If yes, REGISTER YOUR CENTRE! http://diabetologists-abcd.org.uk/n3/ExenatideQW_Audit.htm}

$A B C D$ has launched a nationwide audit of exenatide QW (Bydureon) in the UK to assess real clinical efficacy and safety \& inform future practice and guidelines

- you are invited to submit your patients' anonymised routinely collected data

- using an easy-to-use online tool hosted on the secure NHS network (N3)

- we can provide easy-to-complete paper proformas for use if preferred

- if contributing, you will be able to analyse your local data easily

Please remember: - the more data, the more significant the result will be - all contributors will be listed in publications arising from data submission 